\title{
A THEOREM OF ROLEWICZ'S TYPE IN SOLID FUNCTION SPACES
}

\author{
CONSTANTIN BUŞE \\ Department of Mathematics, West University of Timişoara, Bd. V. Pârvan 4, 1900-Timişoara, România \\ e-mail:buse@hilbert.math.uvt.ro \\ and SEVER S. DRAGOMIR \\ School of Communications and Informatics, Victoria University of Technology, P.O. Box 14428, \\ Melbourne City MC, Victoria 8001, Australia \\ e-mail: sever.dragomir@vu.edu.au
}

(Received 5 May, 2000; revised 23 October, 2000)

\begin{abstract}
Let $\mathbf{R}_{+}$be the set of all non-negative real numbers, $\mathbf{I} \in\left\{\mathbf{R}, \mathbf{R}_{+}\right\}$and $\mathcal{U}_{\mathbf{I}}=\{U(t, s): t \geq s \in I\}$ be a strongly measurable and exponentially bounded evolution family of bounded linear operators acting on a Banach space $X$. Let $\phi: \mathbf{R}_{+} \rightarrow \mathbf{R}_{+}$be a strictly increasing function and $E$ be a normed function space over I satisfying some properties; see Section 2. We prove that if

$$
\phi \circ\left(\chi_{[s, \infty)}(\cdot)\|U(\cdot, s) x\|\right)
$$
\end{abstract}

defines an element of the space $E$ for every $s \in \mathbf{I}$ and all $x \in X$ and if there exists $M>0$ such that

$$
\sup _{s \in \mathbf{I}}\left|\phi \circ\left(\chi_{[s, \infty)}(\cdot)|| U(\cdot, s) x \|\right)\right|_{E}=M<\infty \quad \forall x \in X \text { with }\|x\| \leq 1,
$$

then $\mathcal{U}_{\mathbf{I}}$ is uniformly exponentially stable. In particular if $\psi: \mathbf{R}_{+} \rightarrow \mathbf{R}_{+}$is a nondecreasing function such that $\psi(t)>0$, for all $t>0$, and if there exists $K>0$ such that

$$
\sup _{s \in \mathbf{I}} \int_{s}^{\infty} \psi(\|U(t, s) x\|) d t=K<\infty, \quad \forall x \in X \text { with }\|x\| \leq 1
$$

then $\mathcal{U}_{\mathbf{I}}$ is uniformly exponentially stable. For $\mathbf{I}=\mathbf{R}_{+}, \psi$ continuous and $\mathcal{U}_{\mathbf{R}_{+}}$ strongly continuous this last result is due to $\mathrm{S}$. Rolewicz. Some related results for periodic evolution families are also proved.

2000 Mathematics Subject Classification. 47A30, 93D05, 35B35, 35B40, 46A30.

1. Introduction. Let $\mathbf{T}=\{T(t)\}_{t \geq 0}$ be a strongly continuous semigroup on a Banach space $X$, and $\omega_{0}(\mathbf{T}):=\lim _{t \rightarrow \infty} \frac{\ln [\|T(t)\|]}{t}$ be its growth bound. It is a well known theorem of Datko [9], that if the function $t \mapsto\|T(t) x\|$ belongs to $L^{2}\left(\mathbf{R}_{+}\right)$, for all $x \in X$, then $\omega_{0}(\mathbf{T})$ is negative; i.e. $\mathbf{T}$ is uniformly exponentially stable. This result 
was generalized by Pazy [15] who showed that the exponent $p=2$ may be replaced by $1 \leq p<\infty$, and by Datko [10], who proved the following result.

Let $\mathcal{U}_{\mathbf{R}_{+}}=\{U(t, s): t \geq s \geq 0\}$ be a strongly continuous and exponentially bounded evolution family of bounded linear operators acting on $X$; see definitions below. In what follows we consider that $U(t, s)=0$ if $t<s$. Let us consider the function

$$
t \mapsto U_{s}^{x}(t):=\chi_{[s, \infty)}(t)\|U(t, s) x\|: \mathbf{I} \rightarrow \mathbf{R}_{+}, \quad s \in \mathbf{I}, \quad x \in X .
$$

If there exists $1 \leq p<\infty$ such that $U_{s}^{x}$ belongs to $L^{p}\left(\mathbf{R}_{+}\right)$for all $s \geq 0$ and every $x \in X$ and if, in addition,

$$
\sup _{s \geq 0}\left\|U_{s}^{x}\right\|_{p}=M(x)<\infty \quad \forall x \in X
$$

then the family $\mathcal{U}_{\mathbf{R}_{+}}$is uniformly exponentially stable, that is, there exist the constants $N>0$ and $v>0$ such that

$$
\|U(t, s)\| \leq N e^{-v(t-s)}, \quad \forall t \geq s \geq 0
$$

This last result was generalized by S. Rolewicz [17]. More precisely, S. Rolewicz has proved that if $\psi: \mathbf{R}_{+} \rightarrow \mathbf{R}_{+}$is a continuous and nondecreasing function such that $\psi(t)>0$, for all $t>0, \psi \circ U_{s}^{x}$ belongs to $L^{1}\left(\mathbf{R}_{+}\right)$, for all $s \geq 0$, and if in addition

$$
\sup _{s \geq 0}\left\|\psi \circ U_{s}^{x}\right\|<\infty \quad \forall x \in X \text { with }\|x\| \leq 1
$$

then $\mathcal{U}_{\mathbf{R}_{+}}$is uniformly exponentially stable. See also [18].

A shorter proof of Rolewicz's theorem was given by Q. Zheng [23] (cf. Neerven $[\mathbf{1 4}$, p. 111]) who also removed the continuity assumption about $\psi$. Other proofs of (the semigroup case) Rolewicz's theorem was offered by W. Littman [12], and van Neerven [14, Theorem 3.2.2]. Some related results have been obtained by K.M. Przyłuski [16], G. Weiss [20] and J. Zabczyk [22].

The paper is organized as follows. Section 2 contains the necessary definitions for the paper to be self-contained. In this section we also state the main result. In Section 3 we prove this result and consider some natural consequences. Section 4 is devoted to some dual results connected with a classical result of Barbashin while the last section deals with certain integral characterizations of non-uniform exponential stability.

2. Definitions and notations. Let $X$ be a real or complex Banach space. We shall denote by $\mathcal{L}(X)$ the Banach space of all bounded linear operators acting on $X$. We also denote by $\|\cdot\|$ the norms of vectors and operators in $X$ and $\mathcal{L}(X)$, respectively.

A family $\mathcal{U}_{\mathbf{I}}:=\{U(t, s): t \geq s \in \mathbf{I}\}$ is said to be an evolution family of bounded linear operators on $X$ if and only if

$\left(e_{1}\right) U(t, s) U(s, r)=U(t, r)$ and $U(t, t)=I d$ for all $t \geq r \geq s \in \mathbf{I}$; Id is the identity operator in $\mathcal{L}(X)$.

The evolution family $\mathcal{U}_{\mathbf{I}}$ is said to be 
$\left(e_{2}\right)$ strongly continuous if for every $x \in X$ the function

$$
(t, s) \mapsto U(t, s) x:\{(t, s): t \geq s \in \mathbf{I}\} \rightarrow X
$$

is continuous;

$\left(e_{3}\right)$ strongly measurable if for every $x \in X$ and any $s \in \mathbf{I}$ the function

$$
t \mapsto\|U(t, s) x\|:[s, \infty) \rightarrow \mathbf{R}_{+}
$$

is measurable;

$\left(e_{4}\right)$ exponentially bounded if there are $M_{1} \geq 1$ and $\omega_{1}>0$ such that

$$
\|U(t, s)\| \leq M_{1} e^{\omega_{1}(t-s)} \text { for all } t \geq s \in \mathbf{I}
$$

$\left(e_{5}\right) q$-periodic (with fixed $q>0$ ) if

$$
U(t+q, s+q)=U(t, s) \text { for all } t \geq s \in \mathbf{I} .
$$

It is easy to see that a $q$-periodic and strongly continuous evolution family on $X$ is an exponentially bounded evolution family on $X$ (see for example [4, Lemma 4.1]).

Let $(\mathbf{I}, \mathcal{L}, m)$ be the Lebesgue measure space, and $\mathcal{M}(\mathbf{I})$ be the linear space of all measurable functions $f: \mathbf{I} \rightarrow \mathbf{R}$, identifying the functions which are equal a.e. on $\mathbf{I}$. We consider a function $\rho: \mathcal{M}(\mathbf{I}) \rightarrow[0, \infty]$ with the following properties:

$\left(\mathbf{n}_{1}\right) \rho(f)=0$ if and only if $f=0$;

(n) $\rho(a f)=|a| \rho(f)$ for any scalar $a \in \mathbf{R}$ and any $f \in \mathcal{M}(\mathbf{I})$, with $\rho(f)<\infty$;

$\left(\mathbf{n}_{\mathbf{3}}\right) \rho(f+g) \leq \rho(f)+\rho(g)$ for all $f, g \in \mathcal{M}(\mathbf{I})$.

Let $F=F_{\rho}$ be the set of all $f \in \mathcal{M}(\mathbf{I})$ such that $|f|_{F}:=\rho(f)<\infty$. It is clear that $(F,|\cdot|)$ is a normed linear space. The normed linear subspace $E$ of $F$ is said to be a solid space over I, (see also [19], [21] for similar notions), if the following two conditions hold:

$\left(\mathbf{n}_{4}\right)$ if $f \in E, g \in E$ and $|f| \leq|g|$ a.e., then $|f|_{E} \leq|g|_{E}$;

(n) $\chi_{[0, t]} \in E$ for all $t>0$.

A solid space $E$ over $\mathbf{I}$ has the ideal property if for all $f \in \mathcal{M}(\mathbf{I})$ and any $g \in E$, from $|f| \leq|g|$ a.e. it follows that $f \in E$. It is clear that $F_{\rho}$ has the ideal property.

Let $E$ be a solid space over I. We say that $E$ satisfies the hypothesis $(H)$ if the following condition holds:

(n $\left.\mathbf{n}_{\mathbf{6}}\right)$ if the sequence $\left(A_{n}\right)_{n=0}^{\infty}$ is such that $A_{n} \in \mathcal{L}, m\left(A_{n}\right)<\infty$ and $\chi_{A_{n}} \in E$ then $\left|\chi_{A_{n}}\right|_{E} \rightarrow \infty$ as $n \rightarrow \infty$.

Let $E$ be a solid space. For all $t>0$, we define

$$
\Psi_{E}(t):=\left|\chi_{[0, t]}\right|_{E} \text { and } \Psi_{E}(\infty)=\lim _{t \rightarrow \infty} \Psi_{E}(t) .
$$

It is clear that if $E$ is a solid space that satisfies the hypothesis $(H)$, then $\Psi_{E}(\infty)=\infty$, but the converse statement is not true. See for example [5, Example 1.1]. However if $E$ is rearrangement invariant (see for example [14, p. 222] or [11] for this class of spaces) and $\Psi_{E}(\infty)=\infty$, then $E$ satisfies the hypothesis $(H)$. In this paper we shall prove the following result.

TheOREM 2.1. Let $\phi: \boldsymbol{R}_{+} \rightarrow \boldsymbol{R}_{+}$be a strictly increasing function, $\mathcal{U}_{\mathbf{I}}=\{U(t, s)$ : $t \geq s \in \mathbf{I}\}$ be a strongly measurable and exponentially bounded evolution family of 
bounded linear operators acting on a Banach space $X$ and $E$ be a solid space over $\mathbf{I}$. We suppose that $E$ has the ideal property, $\Psi_{E}(\infty)=\infty$ and

$$
\left|\chi_{[0, t]}\right|_{E} \leq\left|\chi_{[\tau, t+\tau]}\right|_{E} \quad \forall t \geq 0, \forall \tau \in \mathbf{I} .
$$

Here $\chi_{A}$ is the characteristic function of the set $A$. If, for all $x \in X$ and every $s \in \mathbf{I}$, $\phi \circ U_{s}^{x}$ defines an element of the space $E$ and, in addition, there exists $M>0$ such that

$$
\sup _{s \in \mathbf{I}}\left|\phi \circ U_{s}^{x}\right|_{E}=M<\infty \quad \forall x \in X \text { with }\|x\| \leq 1
$$

then $\mathcal{U}_{I}$ is uniformly exponentially stable; i.e., there exist $N>0$ and $v>0$ such that

$$
\|U(t, s)\| \leq N e^{-v(t-s)} \quad(t \geq s \in \mathbf{I}) .
$$

For $E:=L^{p}\left(\mathbf{R}_{+}, \mathbf{C}\right)$ the condition (1) is verified with equality. The condition (1) is essential in the proof of Theorem 2.1; see [5, Example 3.2], but it may hold except in the autonomous case [14, Theorem 3.1.5] and it may also hold except in the periodic case [4, Theorem 4.5]. In the paper [1] the authors replaced the continuity assumptions of solutions, by measurability.

\section{Proof and consequences of Theorem 2.1.}

Proof of Theorem 2.1. We shall prove the Theorem in two steps.

Step 1. Here we shall state that $\mathcal{U}_{\mathbf{I}}$ is uniformly bounded. Upon replacing $\phi$ by some multiple of itself we may assume that $\phi(1)=1$. Also we may assume that $\phi(0)=0$. Let $N$ be a positive integer such that $\left|\chi_{[0, N]}\right|_{E}>M, t_{0} \in \mathbf{I}, t \geq t_{0}+N$ and $x \in X,\|x\| \leq 1$. For $t-N \leq \tau \leq t$ we have

$$
\begin{aligned}
e^{-\omega_{1} N} \chi_{[t-N, t]}(u)\left\|U\left(t, t_{0}\right) x\right\| & \leq e^{-\omega_{1}(t-\tau)} \chi_{[t-N, t]}(u)\|U(t, \tau)\|\left\|U\left(\tau, t_{0}\right) x\right\| \\
& \leq M_{1}\left\|U\left(u, t_{0}\right) x\right\| \quad \forall u \geq t_{0} .
\end{aligned}
$$

Therefore in view of $\left(\mathbf{n}_{\mathbf{4}}\right)$ it follows that:

$$
\left|\phi \circ\left(\frac{1}{M_{1} e^{\omega_{1} N}}|| U\left(t, t_{0}\right) x|| \chi_{[t-N, t]}(\cdot)\right)\right|_{E} \leq\left|\phi \circ U_{t_{0}}^{x}\right|_{E} .
$$

Moreover,

$$
\begin{aligned}
\left|\phi\left(\frac{1}{M_{1} e^{\omega_{1} N}}\left\|U\left(t, t_{0}\right) x\right\|\right) \chi_{[0, N]}(\cdot)\right|_{E} & \leq\left|\phi\left(\frac{1}{M_{1} e^{\omega_{1} N}}|| U\left(t, t_{0}\right) x \|\right) \chi_{[t-N, t]}(\cdot)\right|_{E} \\
& =\left|\phi \circ\left(\frac{1}{M_{1} e^{\omega_{1} N}}|| U\left(t, t_{0}\right) x \| \chi_{[t-N, t]}(\cdot)\right)\right|_{E} .
\end{aligned}
$$

Now from (2) and (4) we have

$$
\phi\left(\frac{1}{M_{1} e^{\omega_{1} N}}|| U\left(t, t_{0}\right) x||\right)\left|\chi_{[0, N]}(\cdot)\right|_{E} \leq M,
$$

and so, using the fact that $\phi(1)=1$, it follows that 


$$
\left\|U\left(t, t_{0}\right) x\right\| \leq M_{1} e^{\omega_{1} N} \text { for all } x \in X \text { with }\|x\| \leq 1 .
$$

Now it is not hard to see that there exists a constant $K_{1}>0$ such that

$$
\sup _{t \geq s \in \mathbf{I}}\|U(t, s)\|=K_{1}<\infty .
$$

Step 2. We consider the function $t \mapsto \Phi(t): \mathbf{R}_{+} \rightarrow \mathbf{R}_{+}$defined by

$$
\Phi(t)= \begin{cases}\int_{0}^{t} \phi(s) d s & \text { if } t<1 \\ 0(t) & \text { if } t \geq 1\end{cases}
$$

It is clear that $\Phi$ is strictly increasing, $\Phi(1)=1$ and $\Phi \leq \phi$. Moreover the inequality (2) from Theorem 2.1 remains valid when we replace $\phi$ by $\Phi$. Let $s \in \mathbf{I}, x \in X$, $\|x\| \leq 1$ and $t>s$. For all $u \geq s$ we have

$$
\begin{aligned}
\chi_{[s, t]}(u)\|U(t, s) x\| & \leq K_{1} \chi_{[s, t]}(u)\|U(u, s) x\| \\
& \leq K_{1}\|U(u, s) x\| .
\end{aligned}
$$

As before, it follows that

$$
\Phi\left(\frac{1}{K_{1}}\|U(t, s) x\|\right) \leq \frac{M}{\left|\chi_{[0, t-s]}\right|_{E}} \quad x \in X,\|x\| \leq 1 .
$$

From (5) for $t-s$ sufficiently large we have the inequality

$$
\|U(t, s)\| \leq K_{1} \Phi^{-1}\left(\frac{M}{\left|\chi_{[0, t-s]}\right|_{E}}\right) .
$$

The proof of Theorem 2.1 is complete if we use the following lemma.

Lemma 3.1. Let $\mathcal{U}_{\boldsymbol{I}}=\{U(t, s): t \geq s \in \boldsymbol{I}\}$ be an exponentially bounded ${ }^{*}$-evolution family of bounded linear operators on a Banach space $X$. If there exists a function $g: \boldsymbol{R}_{+} \rightarrow \boldsymbol{R}_{+}$such that

$$
\inf _{t>0} g(t)<1 \text { and }\|U(t, s)\| \leq g(t-s), \text { for all } t \geq s \in \mathbf{I},
$$

then $\mathcal{U}_{\mathbf{I}}$ is uniformly exponentially stable; that is (3) holds.

For the proof of Lemma 3.1 we refer to [6, Lemma 4].

Corollary 3.2. Let $\phi: \boldsymbol{R}_{+} \rightarrow \boldsymbol{R}_{+}$be a non-decreasing function such that $\phi(t)>0$ for all $t>0$ and $\mathcal{U}_{\mathbf{I}}$ a strongly measurable and exponentially bounded evolution family of bounded linear operators acting on $X$. If there exists a $K>0$ such that

$$
\sup _{s \in \mathbf{I}} \int_{s}^{\infty} \phi(\|U(t, s) x\|) d t=K<\infty, \quad \forall x \in X \text { with }\|x\| \leq 1,
$$

then $\mathcal{U}_{\mathbf{I}}$ is uniformly exponentially stable. 
Proof. This follows from Theorem 2.1 on putting $E=L^{1}\left(\mathbf{I}, \mathbf{R}_{+}\right)$and using the fact that $\phi$ can be replaced by a function $\psi$ which is strictly increasing on $\mathbf{R}_{+}$and $\psi \leq \phi$. Such a function can be defined in the following manner.

Let $\phi(1)=1$ and $a=\int_{0}^{1} \phi(t) d t$. The function

$$
t \mapsto \psi(t):= \begin{cases}\int_{0}^{t} \phi(s) d s & \text { if } t \leq 1, \\ \frac{a t}{a t+1-a} & \text { if } t>1 .\end{cases}
$$

has the desired properties.

Theorem 3.3. Let $\phi: \mathbf{R}_{+} \rightarrow \mathbf{R}_{+}$be a non-decreasing function such that $\phi(t)>0$ for all $t>0, \mathcal{U}_{I}$ a strongly continuous and q-periodic evolution family of bounded linear operators on $X$, and $E$ a solid space over $\mathbf{R}_{+}$that has the ideal property and satisfies the hypothesis $(H)$. If $\phi \circ U_{0}^{x}$ defines an element of the space $E$, for all $x \in X$, then $\mathcal{U}_{\boldsymbol{I}}$ is uniformly exponentially stable.

Proof. It is sufficient to consider the case when $\mathbf{I}=\mathbf{R}_{+}$because if the restriction $\mathcal{U}_{\mathbf{I}}^{0}$ of $\mathcal{U}_{\mathbf{I}}$ to the set $\{(t, s): t \geq s \geq 0\}$ is uniformly exponentially stable then $\mathcal{U}_{\mathbf{I}}$ is uniformly exponentially stable too. We shall modify the first step of Theorem 2.1. The argument is standard, see [15, Theorem 4.4.1], [7, Theorem 2.1], [14, Theorem 2.2] or [5, Theorem 3.1]. In fact we can prove that if $\phi \circ U_{0}^{x}$ defines an element of the space $E$ for some $x \in X,\|x\| \leq 1$ then

$$
\lim _{t \rightarrow \infty}\|U(t, 0) x\|=0
$$

Indeed, if not, then

$$
\limsup _{t \rightarrow \infty}\|U(t, 0) x\|>0
$$

and there exists a $\delta>0$ and a sequence $\left(t_{n}\right)_{n=0}^{\infty}$ with $t_{0}>0$ and $t_{n+1}-t_{n}>\frac{1}{\omega_{1}}$ such that $\left\|U\left(t_{n}, 0\right) x\right\|>\delta$ for all positive integers $n$. Let

$$
J_{n}=\left[t_{n}-\frac{1}{\omega_{1}}, t_{n}\right], A_{n}=\cup_{k=0}^{n} J_{k} \text { and } t \in J_{n} .
$$

We have

$$
\phi(\delta) \leq \phi\left(\left\|U\left(t_{n}, 0\right) x\right\|\right) \leq \phi\left(M_{1} e\|U(t, 0) x\|\right) .
$$

Therefore, as $\phi$ can be considered strictly increasing, it follows that

$$
\delta \leq M_{1} e\|U(t, 0) x\| \quad \forall t \in A_{n}, \forall n \in \mathbf{N} .
$$

Now in view of hypothesis $(H)$ we have

$$
\infty=\lim _{n \rightarrow \infty} \phi\left(\frac{\delta}{M_{1} e}\right)\left|\chi_{A_{n}}(\cdot)\right|_{E} \leq\left|\phi \circ U_{0}^{x}\right|_{E},
$$


which is a contradiction. Using the linearity of $U(t, 0)$ and the uniform boundedness principle it follows that there exists a constant $K_{2}>0$ such that

$$
\sup _{t \geq 0}\|U(t, 0)\|=K_{2}<\infty
$$

Moreover in view of $\left(\mathbf{e}_{\mathbf{4}}\right)$ and $\left(\mathbf{e}_{5}\right)$ it easily follows from, for example [5, Proof of Theorem 3.1] that

$$
\sup _{t \geq s \geq 0}\|U(t, s)\| \leq K_{2} M_{1} e^{\omega_{1} q}<\infty
$$

From here the proof can be continued as in the proof of Theorem 2.1.

4. The dual results. A reformulation of an old result of E. A. Barbashin [2, Theorem 5.1] is as follows.

Let $\mathcal{U}_{\mathbf{R}_{+}}$be an exponentially bounded evolution family of bounded linear operators on $X$. We suppose that the function

$$
s \mapsto\|U(t, s)\|:[0, t] \rightarrow \mathbf{R}_{+}
$$

is measurable, for all $t>0$. If

$$
\sup _{t \geq 0} \int_{0}^{t}\|U(t, s)\| d s<\infty,
$$

then $\mathcal{U}_{\mathbf{R}_{+}}$is uniformly exponentially stable.

See also [13] and [3] for similar facts.

The following theorem is a generalization of the result above in the case $\mathbf{I}=\mathbf{R}$.

Theorem 4.1. Let $\phi: \boldsymbol{R}_{+} \rightarrow \boldsymbol{R}_{+}$be a non-decreasing function such that $\phi(t)>0$ for all $t>0$ and $\mathcal{U}_{\mathbf{R}}=\{U(t, s): t \geq s\}$ an exponentially bounded evolution family of bounded linear operators on $X$. We assume that the function

$$
s \mapsto\|U(t, s)\|:(-\infty, t] \rightarrow \mathbf{R}_{+}
$$

is measurable, for all $t \in \mathbf{R}$. If

$$
\sup _{t \in \mathbf{R}} \int_{-\infty}^{t} \phi(\|U(t, s)\|) d s<\infty,
$$

then $\mathcal{U}_{\mathbf{R}}$ is uniformly exponentially stable.

Proof. Let $X^{*}$ be the dual space of $X$ and $U(t, s)^{*}$ the adjunct operator of $U(t, s)$ for $t \geq s$. Let $t \in \mathbf{R}, u=-t$ and 


$$
V(s, u):=U(-u,-s)^{*} \in \mathcal{L}\left(X^{*}\right) .
$$

We have

$$
\begin{aligned}
\int_{-\infty}^{t} \phi(\|U(t, s)\|) d s & =\int_{-\infty}^{t} \phi\left(\left\|U(t, s)^{*}\right\|\right) d s \\
& =\int_{-t}^{\infty} \phi\left(\left\|U(t,-s)^{*}\right\|\right) d s \\
& =\int_{u}^{\infty} \phi\left(\left\|U(-u,-s)^{*}\right\|\right) d s \\
& =\int_{u}^{\infty} \phi(\|V(s, u)\|) d s .
\end{aligned}
$$

It is clear that the family $\mathcal{V}_{\mathbf{R}}:=\{V(s, u): s \geq u \in \mathbf{R}\}$ is an exponentially bounded evolution family of bounded linear operators on $X^{*}$ and, in addition, the function

$$
s \mapsto\|V(s, u)\|:[u, \infty) \rightarrow \mathbf{R}_{+}
$$

is measurable, for all $u \in \mathbf{R}$.

From the uniform variant of Corollary 3.2 it follows that $\mathcal{V}_{\mathbf{R}}$ is uniformly exponentially stable. Hence $\mathcal{U}_{\mathbf{R}}$ is uniformly exponentially stable, too.

Theorem 4.2. Let $\phi: \mathbf{R}_{+} \rightarrow \mathbf{R}_{+}$be a non-decreasing function such that $\phi(t)>0$ for all $t>0$, and $\mathcal{U}_{\boldsymbol{R}}$ a q-periodic evolution family of bounded linear operators on $X$. We assume that the function

$$
t \mapsto\|U(0,-t)\|:[0, \infty) \rightarrow \mathbf{R}_{+}
$$

is measurable. If

$$
\int_{0}^{\infty} \phi(\|U(0,-t)\|) d t<\infty
$$

then $\mathcal{U}_{\mathbf{R}}$ is uniformly exponentially stable.

Proof. As in the proof of Theorem 4.1 it follows that

$$
\int_{0}^{\infty} \phi(\|V(t, 0)\|) d t<\infty .
$$

Now apply Theorem 3.3 for $E=L^{1}\left(\mathbf{R}_{+}\right)$. 
COROLlary 4.3. Let $\phi$ and $\mathcal{U}_{\mathbf{R}}$ be as in Theorem 4.2. We shall assume that the function

$$
s \mapsto|| U(t, s) \|:[0, t] \rightarrow \mathbf{R}_{+}
$$

is measurable on $[0, t]$ for all $t>0$. If

$$
\sup _{t \geq 0} \int_{0}^{t} \phi(\|U(t, s)\|) d s=N_{0}<\infty,
$$

then $\mathcal{U}_{\mathbf{R}}$ is uniformly exponentially stable.

Proof. From (6) for $t=n q, n \in \mathbf{N}$ it follows that

$$
\begin{aligned}
N_{0} & \geq \sup _{n \in \mathbf{N}} \int_{0}^{n q} \phi(\|U(n q, s)\|) d s=\sup _{n \in \mathbf{N}} \int_{0}^{n q} \phi(\|U(0, s-n q)\|) d s \\
& =\sup _{n \in \mathbf{N}} \int_{0}^{n q} \phi(\|U(0,-t)\|) d t=\int_{0}^{\infty} \phi(\|U(0,-t)\|) d t .
\end{aligned}
$$

Now we can apply Theorem 4.2 to complete the proof.

5. Nonuniform exponential stability. An evolution family $\mathcal{U}_{\mathbf{I}}=\{U(t, s)$ : $t \geq s \in \mathbf{I}\}$ of bounded linear operators on $X$ is said to be exponentially stable if there exists a constant $v>0$ and a function $N: \mathbf{I} \rightarrow(0, \infty)$ such that

$$
\|U(t, s)\| \leq N(s) e^{-v(t-s)} \quad \forall t \geq s \in \mathbf{I} .
$$

It is easy to see that the function $N(\cdot)$ can be chosen to be non-decreasing on I. In the case $\mathbf{I}=\mathbf{R}_{+}$we have the following Datko's theorem version for non-uniform exponential stability.

THEOREm 5.1. A strongly continuous and exponentially bounded evolution family $\mathcal{U}_{\boldsymbol{R}_{+}}=\{U(t, s): t \geq s \geq 0\}$ is exponentially stable if and only if there exists an $\alpha>0$ such that

$$
\int_{s}^{\infty} e^{\alpha t}\|U(t, s) x\| d t<\infty \quad \forall x \in X \text { and } \forall s \geq 0 .
$$

For the proof of Theorem 5.1 and its other variants we refer to [8, Theorem 2.1], [7, Theorem 2.2] or [5, Theorem 3.2]. The extension of Theorem 5.1 for the case $\mathbf{I}=\mathbf{R}$ can be easily obtained. Moreover we have the following result.

TheORem 5.2. Let $\phi: \mathbf{R}_{+} \rightarrow \mathbf{R}_{+}$be a non-decreasing function such that $\phi(t)>0$, for all $t>0$, and $\mathcal{U}_{\mathbf{I}}$ a strongly measurable and exponentially bounded evolution family of bounded linear operators on $X$. If there exists an $\alpha>0$ such that 


$$
\int_{s}^{\infty} \phi\left(e^{\alpha t}\|U(t, s) x\|\right) d t<\infty \quad \forall s \in \mathbf{I} \text { and } \forall x \in X,
$$

then $\mathcal{U}_{\mathbf{I}}$ is exponentially stable.

The proof of Theorem 5.2 follows as in [7, Theorem 2.2]. The Barbashin's theorem version for exponential stability is as follows.

Theorem 5.3. Let $\phi: \mathbf{R}_{+} \rightarrow \mathbf{R}_{+}$be a non-decreasing function such that $\phi(t)>0$ for all $t>0$ and $\mathcal{U}_{\mathbf{R}}$ an exponentially bounded evolution family of bounded linear operators on $X$. We assume that the function

$$
s \mapsto\|U(t, s)\|:(-\infty, t] \rightarrow \mathbf{R}_{+}
$$

is measurable, for all $t \in \mathbf{R}$. If there exists an $\alpha>0$ such that

$$
\int_{-\infty}^{t} \phi\left(e^{-\alpha s}\|U(t, s)\|\right) d s<\infty \quad \forall t \in \mathbf{R},
$$

then $\mathcal{U}_{\mathbf{R}}$ is exponentially stable.

Proof. As in the Proof of Theorem 4.1, it follows that the family $\mathcal{V}_{\mathbf{R}}:=\{V(s, u)$ : $s \geq u \in \mathbf{R}\}$, where $V(s, u):=U(-u,-s)^{*}$, is exponentially stable; that is, there exist $v>0$ and a function $N: \mathbf{R} \rightarrow(0, \infty)$ such that

$$
\|V(s, u)\| \leq N(u) e^{-v(s-u)} \quad \forall s \geq u \in \mathbf{R} .
$$

Let $\alpha:=-u \geq \beta:=-s$. Then

$$
\|U(\alpha, \beta)\| \leq N(-\alpha) e^{-v(\alpha-\beta)} \leq N(-\beta) e^{-v(\alpha-\beta)} ;
$$

that is, $\mathcal{U}_{\mathbf{R}}$ is exponentially stable.

ACKNOWLeDGements. The authors would like to thank the anonymous referees for their comments and suggestions on preliminary versions of this paper, which have led to a substantial improvement in its readability.

\section{REFERENCES}

1. D. D. Bainov and S. I. Konstantinov, Exponential stability of the solutions of linear homogeneous differential equations with impulse effect in Banach spaces, Dokl. Bulg. Acad. Sc. 40 (1987), 29-30.

2. E. A. Barbashin, Introduction in the theory of stability (Izd. Nauka, Moscow, 1967), (Russian).

3. C. Buşe and M. Giurgiulescu, A new proof for a Barbashin's theorem in the periodic case, preprint in Evolution equations and semigroups, (http:// malserv.mathematik.unikarlsruhe.de/evolve-1/index.html).

4. C. Buşe and A. Pogan, Individual Exponential Stability for *-Evolution Families of Linear and Bounded Operators, New Zealand J. Math. 30 (2001), 15-24. 
5. C. Buşe, Asymptotic stability of evolutors and normed function spaces, Rend. Sem. Mat. Univ. Pol. Torino 55, 2(1997), 109-122.

6. C. Buşe, On the Perron-Bellman theorem for evolutionary processes with exponential growth in Banach spaces, New Zealand J. Math. 27 (1998), 183-190.

7. C. Buşe, Nonuniform exponential stability and Orlicz functions, Comm. Math. Prace Matematyczne 36 (1996), 39-47.

8. C. Buşe, On nonuniform exponential stability of evolutionary processes, Rend. Sem. Univ. Pol. Torino 52 4(1994), 395-406.

9. R. Datko, Extending a theorem of A.M. Liapunov to Hilbert space, J. Math. Anal. Appl. 32 (1970), 610-616.

10. R. Datko, Uniform asymptotic stability of evolutionary processes in a Banach space, SIAM J. Math. Analysis 3 (1973), 428-445.

11. S. G. Krein, Yu. I. Petunin and E. M. Semeonov, Interpolation of linear operators, Transl. Math. Monogr. 54 (Amer. Math. Soc., Providence, 1982).

12. W. Littman, A generalization of a theorem of Datko and Pazy, in Lecture Notes in Control and Information Science No. 130 (Springer-Verlag, 1989), 318-323.

13. M. Megan, Proprietes qualitatives des systems lineaires controles dans les espaces de dimension infinie, Monographies Mathematiques (Timisoara, 1988).

14. J. M. A. M. van Neerven, The asymptotic behaviour of semigroups of linear operators (Birkhäuser Verlag, 1996).

15. A. Pazy, Semigroups of linear operators and applications to partial differential equations (Springer-Verlag, 1983).

16. K. M. Przyłuski, On a discrete time version of a problem of A. J. Pritchard and J. Zabczyk, Proc. Roy. Soc. Edinburgh, Sect A 101 (1985), 159-161.

17. S. Rolewicz, On uniform N-equistability, J. Math. Anal. Appl. 115 (1986), 434-441.

18. S. Rolewicz, Functional analysis and control theory (D. Reidel and PWN-Polish Scientific Publishers, Dordrecht-Warszawa, 1987).

19. S. Rolewicz, Metric linear spaces, 2nd ed. (D. Reidel and PWN-Polish Scientific Publishers, Dordrecht-Warszawa, 1985).

20. G. Weiss, Weakly $l^{p}$-stable linear operators are power stable, Int. J. Systems Sci. 20 (1989), 2383-2328.

21. A. C. Zaanen, Integration, 2nd ed. (North Holland, 1967).

22. A. Zabczyk, Remarks on the control of discrete-time distributed parameter systems, SIAM J. Control 12 (1974), 731-735.

23. Q. Zheng, The exponential stability and the perturbation problem of linear evolution systems in Banach spaces, J. Sichuan Univ. 25 (1988), 401-411 (in Chinese). 